\title{
Structure of polyelectrolyte brushes studied by coarse grain simulations
}

\author{
Hitoshi WASHIZU ${ }^{1,2, *}$, Tomoyuki KINJO ${ }^{1,2}$, Hiroaki YOSHIDA ${ }^{1,2}$ \\ ${ }^{1}$ Toyota Central R\&D Labs., Inc., 41-1 Yokomichi, Nagakute, Aichi 480-1192, Japan \\ ${ }^{2}$ Elements Strategy Initiative for Catalysts and Batteries, Kyoto University, Katsura, Kyoto 615-8520, Japan \\ Received: 21 September 2013 / Revised: 13 February 2014 / Accepted: 14 February 2014 \\ (C) The author(s) 2014. This article is published with open access at Springerlink.com
}

\begin{abstract}
As an example of a very low friction system, Monte Carlo Brownian dynamics simulations have been used to calculate equilibrium structures of a polyelectrolyte brush grafted onto planes. The polymers were calculated in a semi-flexible coarse-grain model that is appropriate to treat the charge density of the polyion. The effect of linear charge density on the polyion $\xi$, the surface negative charge, and added salts were studied. In salt-free solution, scaling theories predicted the structure well in the low $\xi$ region. In the high $\xi$ region, additional shrinkage was found from the theories due to counterion condensation. The effect of surface charge showed not only the repulsion of the polyion from the surface but also the shrinkage in the high $\xi$ region due to the additional counterions required for electrical neutrality. The addition of salts led to the shrinkage of the brush heights, and in the high $\xi$ region, additional extension was found. The computational strategy for calculating the friction dynamics of the system is also discussed.
\end{abstract}

Keywords: polyelectrolyte brush; friction; Monte Carlo Brownian dynamics simulation; soft materials; automotive tribology

\section{Introduction}

One of the most useful applications of soft materials in automotive technology is the use of lubricants used in machine elements. The theoretical treatment of lubricants, however, is difficult even if one only considers a single stroke of an internal combustion engine. This complexity arises because the hydrodynamic lubrication and boundary lubrication regime, which are created by the velocity of the piston motion, have very different physico-chemical origins. Molecular simulations provide a suitable analytical tool to understand the soft nature of lubricants because ad-hoc parameters are not required, except for a set of interatom or molecular interactions. In a case of

* Corresponding author: Hitoshi WASHIZU.

E-mail: h@washizu.org

A preliminary version of this work was presented at the $3^{\text {rd }}$ International Symposium on Tribology of IFToMM, Luleå, Sweden, 2013. elastohydrodynamic lubrication, molecular dynamics simulations have been used to study the mechanism of momentum transfer related to the molecular structure of hydrocarbon fluids, the phase transition of the fluids under high pressure, and the submicron thickness behavior of an oil film [1]. For boundary lubrication, the mechanism behind the low friction of the diamond-like carbon containing silicon (DLC-Si) coating was understood by conducting a molecular dynamics study of an adsorbed boundary water film [2]. Recently, the ultra-low mechanisms of solid layered graphites have also been solved by coarsegrain molecular simulation, which is normally used to model soft materials such as polymers or liquid crystals [3]. The origin of solid friction itself is the phonon dissipation generated in deep bulk solid atoms [4-6]; the friction of layered materials is understood in terms of the thermal motion of a transferred pile of graphene sheets.

The strongest force, and also the one with longest 
range at the tribological interface, is the long-range Coulomb interaction, which is the force between the ions [7]. The flexibility and high functions of biological phenomena are the result of this force. The ultimate goal of controlling friction in automotive tribology may be linked to the control of forces in the ion atmosphere. Synovial joints represent a typical biomimetic system in tribology that is governed by this force. In fact, the tribological properties of polyelectrolyte brushes have been widely studied as model systems of synovial joints, which show very low friction [8-10]. In the work described here, some preliminary results of computer simulations related to the friction of polyelectrolyte brushes are presented, and the strategy of simulations to understand the phenomena is discussed.

The theoretical understanding of the friction mechanism of the polyelectrolyte brush is extremely difficult even when a simple model is used for polyion molecules. This complexity arises because the segment motion of the polyion chain, the concentrations of small mobile ions, the long range Coulomb potential, and the solvent velocity are coupled with each other through essentially nonlinear equations. The ion environment around a polyion is described as follows. The high charge density that is present in many biopolymers means that the counterions condense in the vicinity of a polyion. The condensed layer surrounds a diffuse ion layer of counter- and co-ions, which can be treated by the Debye-Hückel approximation. The diffuse ion layer is surrounded by bulk salt. The static and the dynamic behavior of the three layers in the ion environment is different [11].

Coarse-grain simulations represent a useful tool to analyze the mechanism for the low friction of polyelectrolyte brushes $[12,13]$. In these simulations, the solvent molecules are treated explicitly and this requires significant computational resources. The importance of the system may be due to the nonuniformity of the small ion distribution, with these ions interacting with each other throughout the whole space of the solution. On the other hand, if the motion of solvent molecules is neglected [14], the effect of solvent flow, which may play a significant role, cannot be incorporated. Furthermore, the important effect of solvent hydration, which is suggested by experimental results [9], cannot be discussed.
A simulation that involves coupling the dynamics of a vast ion atmosphere, the effect of local solvation around each of the ions, and macroscopic friction should be constructed to overcome the aforementioned difficulties. Multiphysical coarse-grain simulators for molecular-scale to meso-scale dynamics were constructed. In this study, the effect of long-range Coulomb interactions on the structure of the brushes is discussed.

The Monte Carlo Brownian dynamics method [15] was used to simulate the nature of polyelectrolyte brushes. To gain a basic understanding, the effect of salt on the structure of polyelectrolyte brushes was studied. Polyions were grafted onto the surface at an equal pitch. Long-range Coulomb interactions were coarse grained by the modified particle-particle particle-cell method. For a flexible polyion model of 128 segments, a bond and bond angle degree of freedom was adopted. The linear charge densities of the polyions are an essential factor in the friction of a polyelectrolyte brush [16].

To understand the charge density effect, the linear charge density parameter of the polyion was set to 0.5, 1.0, 2.0 and 4.0 to discuss Manning's counterion condensation theory [17]. The thermal equilibrium structures at room temperature of brush systems in salt-free solution, the effect of surface charge, and the effect of the added salt were studied.

\section{Model}

To discuss the effect of the linear charge density $\xi$ on the structure of a polyelectrolyte brush, the polyelectrolyte molecules were modeled as follows. Since the results are mainly compared with analytical theories, the polyelectrolyte model was set to a simple linear semi-flexible chain model. According to Manning's counterion condensation theory, $\xi$ is defined as the charge density of the polyion using the Bjerrum length $l_{\mathrm{B}}$ of the system and the charge spacing $b$ in the longitudinal direction of a linear polyion by $\xi=l_{\mathrm{B}} / b$, where the Bjerrum length $l_{B}$ is the length at which thermal energy and electrostatic energy are comparable, $l_{\mathrm{B}}=e^{2} / 4 \pi \varepsilon_{0} \varepsilon k_{\mathrm{B}} T$, where $T=$ $298 \mathrm{~K}$ is the temperature, $k_{\mathrm{B}}$ is the Boltzmann constant, $e$ is the unit charge, and $\varepsilon_{0}$ and $\varepsilon$ are the permittivity 
and the relative permittivity, respectively. The solvent was treated as a dielectric continuum with the relative permittivity of pure water at $298 \mathrm{~K}(\varepsilon=78.3)$.

A monomer fragment of a flexible polyelectrolyte with $N=128$ was modeled by a sequence of hard core beads of $0.06 \mathrm{~nm}$ radius. The bond and bond angle energies were calculated for the intramolecular interaction. The bond interaction energy $U_{\text {bond }}$ between two neighboring beads at a distance $r$ was calculated by $U_{\text {bond }}=k_{\mathrm{b}}\left(r-r_{0}\right)^{2}$, where $r_{0}=0.12 \mathrm{~nm}$, i.e., the average distance between two beads and $k_{\mathrm{b}}=100 k_{\mathrm{B}} T$ is the bond constant. The bond angle interaction energy $U_{\text {angle }}$ between three neighboring beads at an angle $\theta$ was calculated by $U_{\text {angle }}=k_{\mathrm{a}}\left(\theta-\theta_{0}\right)^{2}$, where $\theta_{0}=120$ degrees, i.e., the average angle between three beads, and $k_{\mathrm{a}}=1 k_{\mathrm{B}} T$ is the bond angle constant. These parameters were used to set a polyion of $\xi=1.0$ by placing negative charges in the middle of every 4 beads when the beads are stretched into a linear shape with their equilibrium distances and angles maintained. In the same way, $\xi=0.5,2.0,4.0$ were set by placing negative charges in the middle of every 8,2 , and 1 beads, respectively. The hydrated univalent counter- and co-ions were modeled as hard spheres of $0.15 \mathrm{~nm}$ radius.

The Monte Carlo (MC) cell is a rectangle of $L_{x y}=$ $2 l_{x y}, 4 l_{x y}, 8 l_{x y}, l_{x y}=8.76 \mathrm{~nm}$ in the $x, y$ direction and $L_{z}=35.0 \mathrm{~nm}$ in the $z$ direction. Four polyions were placed at the bottom of the simulation cell with equal spacing, i.e., the polyions were tied by the beads, which in turn were fixed at $x, y= \pm 1 / 4 L_{x, y}, z=0$.

\section{Simulation}

The conventional Metropolis MC procedure was used to generate a canonical ensemble. The configuration energy was calculated as the sum of electrostatic interactions and intermolecular interactions.

Electrostatic interactions were coarse-grained by the modified particle-particle particle-cell method [18]. Interactions were periodically calculated in the $x, y$ directions. The ion pair energy in the vicinity of $x, y, z<1 / 9 L_{z}$ was calculated directly. An $\Omega^{-1}$ value of $0.2 \mathrm{~nm}$ was used as the maximum ion displacement allowed for an MC move along each coordinate direction.
The new configuration generated by a random move of a single ion was accepted or rejected according to the probability $\min \left\{1, \exp \left(-\Delta U / k_{\mathrm{B}} T\right)\right\}$, where $U$ is the change in the configurational energy that would result from the move. Periodical boundary conditions were used in the $x, y$ directions and a reflective boundary condition was used in the $z$ direction.

Although the Metropolis time scale $t$ was fixed by the relation $6 d \Delta t \Omega^{2}=1$, where $d$ is the diffusion coefficient for the small ion species in water at infinite dilution [15,3], it was not necessary to relate the Metropolis and physical time scales in this work as only time averages were calculated.

In the salt-free solution, $16 \times 4,32 \times 4,64 \times 4,128 \times 4$ counterions were included when $\xi=0.5,1,2,4$, respectively. In the salt solution, 512 (when $L_{x y}=2 l_{x y}$ ) and 1024 (when $L_{x y}=4 l_{x y}$ ) pairs of counter- and co-ions were included as added salt. The equilibrium distribution of polyions and counter- and co-ions was obtained after $1.5 \times 10^{7} \mathrm{MC}$ steps were run from a random initial distribution.

\section{Results and discussion}

\subsection{Structure of the brush in salt-free solution}

The average heights $h$ of polyions in salt-free solutions are plotted in Fig. 1. Two definitions of heights are plotted, namely the mean height of the open end monomer to the substrate surface, $\left\langle z_{\text {end }}\right\rangle$, and the mean maximum height of the monomers along the

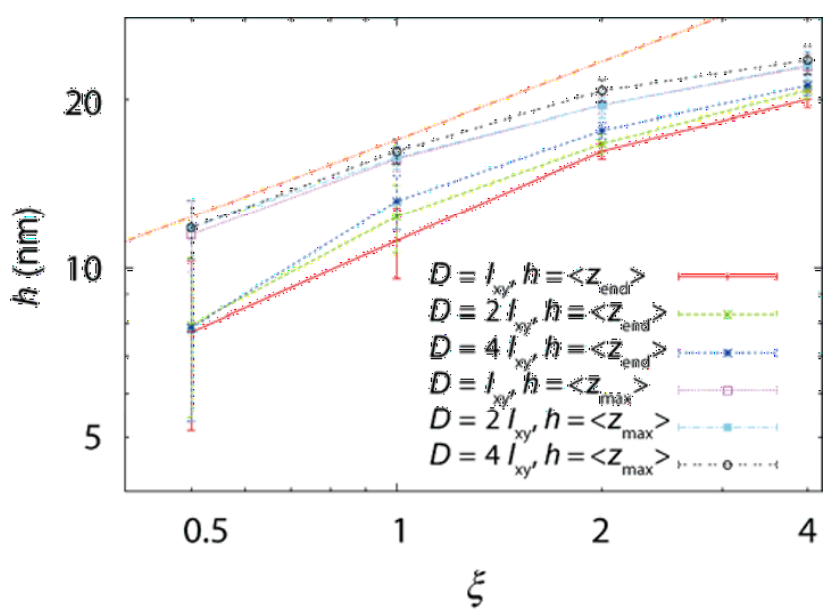

Fig. 1 Dependence of the equilibrium average heights $h$ of polyions on linear charge densities $\xi$ in salt-free solutions. 
chain, $<z_{\max }>$. In both definitions of heights, polyions are stretched on increasing the polymer charge density $\xi$, thus reflecting the increase in the electrostatic repulsion of charged sites. The difference between $<z_{\text {end }}>$ and $\left\langle z_{\max }\right\rangle$ is large when $\xi$ are small and the difference becomes less marked when $\xi$ are large. This trend is related to the shape of the polyions. Snapshots of polyion solutions for $\xi=0.5$ and $\xi=4.0$ are shown in Fig. 2. The open end monomers are plotted as triangles. In the stretched polyions for $\xi=4.0$, the open end monomers are almost at the top of the monomer distribution, whereas in the case where $\xi=0.5$ the open end monomer is located almost in the middle of the monomer distribution. A comparison of our results with those of scaling theories [19-22] is discussed below. The straight line plotted in Fig. 1 shows a power low fit in $h=<z_{\max }>$ and the surface polymer spacing $D=l_{x y}$. According to the scaling theory of Pincus [19], the brush thickness is determined from the balance between the osmotic pressure and the chain elasticity. In the osmotic regime, for the case with high grafting density and low activity of added salt or salt-free conditions, the thickness is independent of the grafting density and the brush height $h$ behaves as $h \approx N a \xi^{\alpha}, \alpha=1 / 2$, where $a$ is
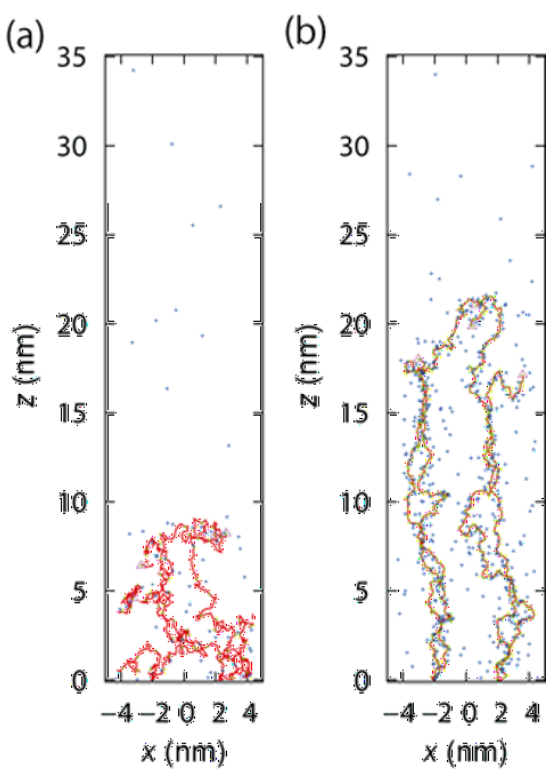

Fig. 2 Snapshots of polyelectrolyte brush solutions. (a) Salt-free solution with $\xi=0.5$. (b) Salt-free solution with $\xi=4.0$. $D=l_{x y}$. Red line, polyion; yellow/green circles, charge on polyion; blue cross, counterions; pink triangle, open end monomers. the monomer size. Zhulina et al. [22] found that the scaling relation in the osmotic regime changes to $h \approx N a \xi^{\alpha}, \alpha=2 / 5$ owing to excluded volume effects. Our numerical result $0.42 \leqslant \alpha \leqslant 0.46$ is in good agreement with these theories. The scaling theories do not treat the high polymer charge density region of $\xi \geqslant 1$, since counterion condensation occurs in this region. In the $\xi \geqslant 1$ region, the scaling relation break and $h$ are lower than those obtained with the scaling law. This situation arises because counterions condense on the polyion surface to obtain the same thermal stability as for $\xi=1$, meaning that the condensed counterions do not contribute to the counterion osmotic pressure.

The effect of polyion spacing $D$, i.e., the distance between two anchoring points $\left(\sigma=a^{2} / D^{2}\right.$ is the surface grafting density), is also plotted in Fig. 1. Although our results show that the brush height $h$ depends weakly on the grafting density, a slight decrease in brush height $h$ with grafting density was found. In some theories, such as mean field calculation, $h$ increases with grafting density [22], whereas a recent molecular dynamics simulation [23] showed the same trend as our results. To consider the mechanism, monomer distributions and counterion distributions for different grafting densities are plotted in Fig. 3. Each distribution shows depletion from the surface in the vicinity of the surface. Comparison of the monomer distributions in Figs. 3(a), 3(b) and 3(c) shows that polyions come into contact with one another in Fig. 3(a) but they are completely separated in Fig. 3(c) and the intermediate situation is observed in Fig. 3(b). The relative distribution of counterions shows two phases in Figs. 3(e) and 3(f), i.e., counterions outside the polyion region and counterions inside the polyion region. More precisely, due to the counterion condensation for $\xi=4.0$, counterions inside the polyion region can be distinguished from those in the condensed layer and diffuse layer, although this difference does not need to be considered. According to Oosawa [24], the extensive force of spherical macroions is considered in this two phase model. The extensive force due to Coulomb repulsion between charged groups $F_{1}$ is $F_{1} \approx n^{* 2}$, where $n^{*}$ is the effective charge in the volume of polyion. In salt-free solution $n^{*}=n-n^{\prime}$, where $n$ is the charge of the polyion, 
(a)

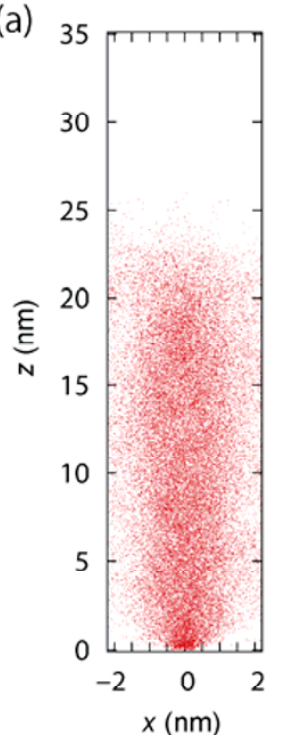

(d)

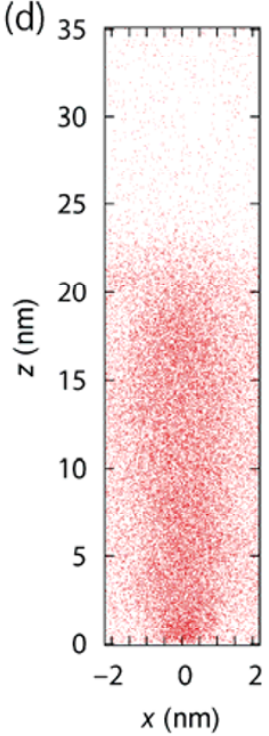

(b)

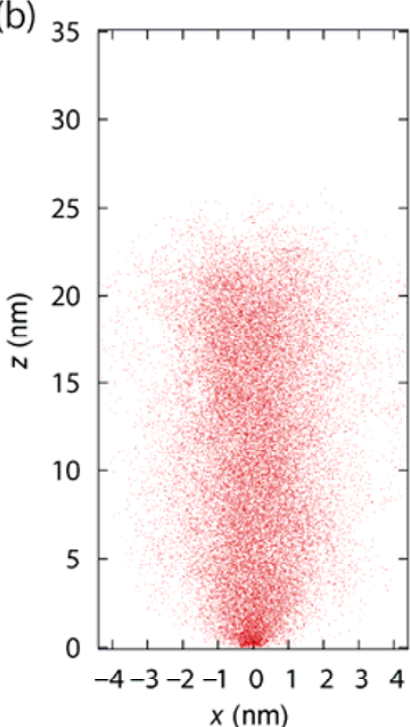

$(\mathrm{e}) 35$

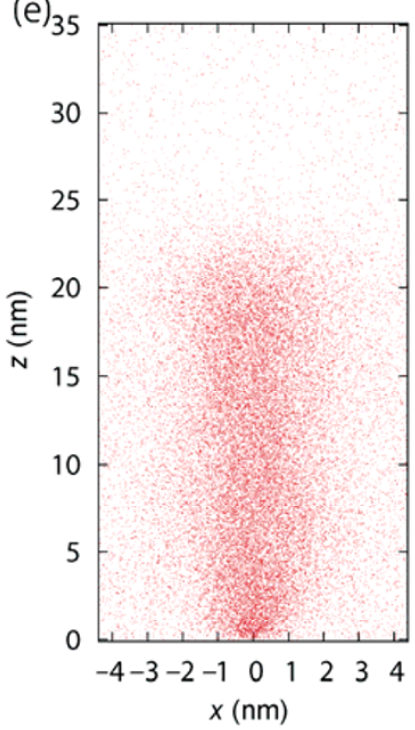

(c) 35

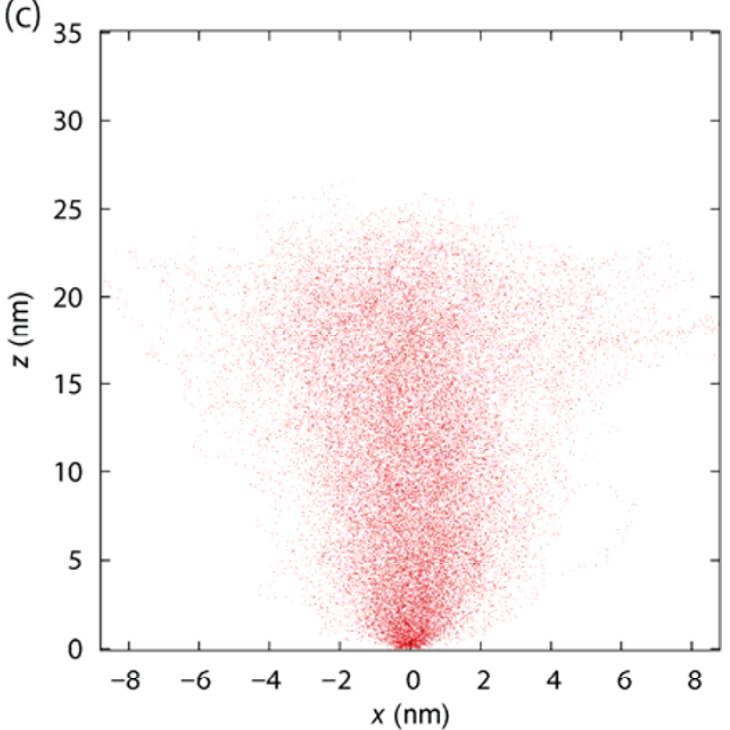

(f) 35

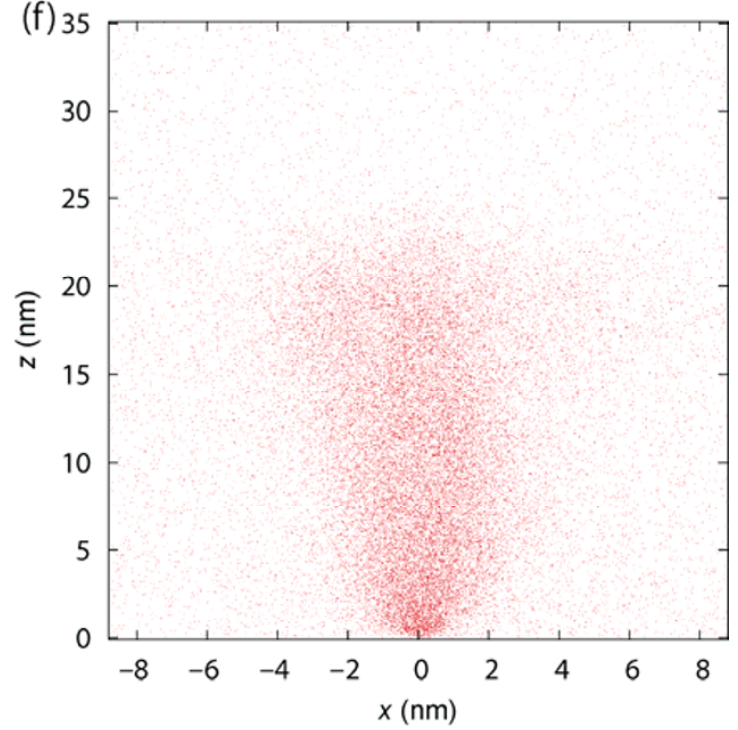

Fig. 3 Monomer distributions (a, b, c) and counterion distributions (d, e, f) of salt-free solutions for $\xi=4.0$. (a, d) $D=l_{x y}$, (b, e) $D=2 l_{x y}$, (c, f) $D=4 l_{x y}$.

which is equal to the number of total counterions, and $n^{\prime}$ is the number of the bound counterions in the volume of the polyion. The osmotic force $F_{2} \approx(1-\gamma)$ also acts on the system, where $\gamma$ is the activity coefficient and the total extensive force is $F_{1}+F_{2}$. In the spherical macroion the apparent charge $n^{*}$ decreases with increasing concentration. The activity coefficient $\gamma$ also increases with increasing concentration and thus the total extensive force decreases with increasing concentration. If we apply this situation to the grafting of polyelectrolytes, the extension force increases slightly on decreasing the grafting density.
A more detailed discussion concerning the comparison with other scaling theories will be addressed in a future publication.

\subsection{Effect of surface charge on the structure of the brush}

Due to the low surface roughness, many friction tests on polyelectrolyte brushes are carried out on the surface of mica $[8,9]$. Mica has a negative charge per $0.48 \mathrm{~nm}^{2}$ or 2.08 negative charges per $\mathrm{nm}^{2}$ [25]. To discuss the charge effect on the surface, the polyelectrolyte brush system was simulated with surface charge. The surface 
charge was spread randomly at a density of $2.0 \mathrm{e} / \mathrm{nm}^{2}$ and the same number of counterions were added to achieve electrical neutrality. The average heights $h$ of polyions in salt-free solutions with and without surface charge are plotted in Fig. 4 . The negatively charged surface does not simply contribute to the extension of the negatively charged polyions. An extension effect, i.e., depletion from the surface, was found in the low $\xi$ region, whereas a shrinkage effect was found in the high $\xi$ region. To facilitate a discussion of this result, the counterion distribution is plotted in Fig. 5. A densely distributed counterion layer was found on the surface due to the relatively high surface charge density of mica. The negative charge on the surface is not completely compensated by this densely distributed area and a Debye-Huckel-type ion atmosphere region exists above the densely distributed area. In the Debye-Huckel-type ion atmosphere, negative charges on the polyion experience a repulsive force directly from the surface. For this reason, the extension effect is found in the low $\xi$ region, where most of the negative charges on the polyion are distributed in the ion atmosphere belonging to the surface charge. In the high $\xi$ region, the direct effect from the surface is weak due to the stretched shape of the polyion. In this case the counterion added to achieve electrical neutrality acts as the added salt. For the case shown in Fig. 5, if one considers the pair of cation and anion for the surface charge as the added salt, the added salt concentration corresponds to $95 \mathrm{mM}$, which is sufficient to change the structure.

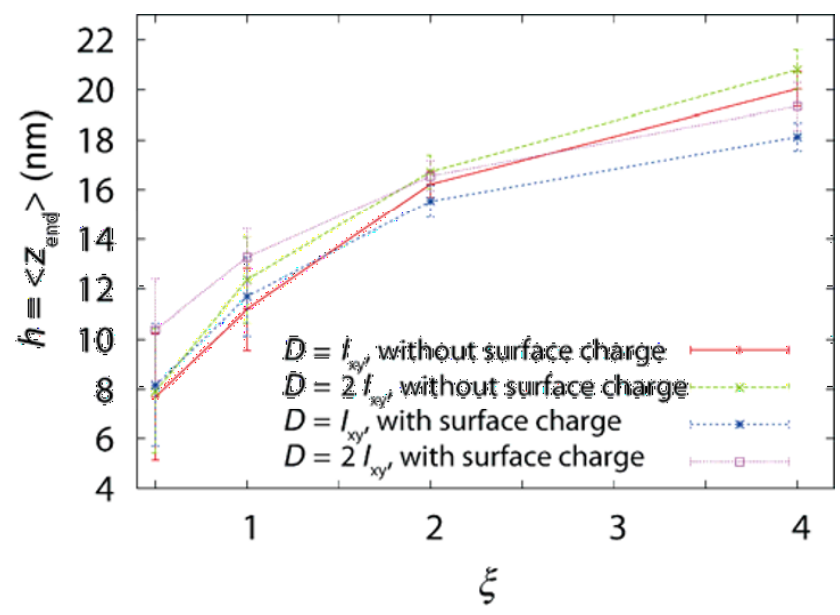

Fig. 4 Dependence of equilibrium average heights $\mathrm{h}$ of polyions on linear charge densities $\xi$ with and without surface charge.
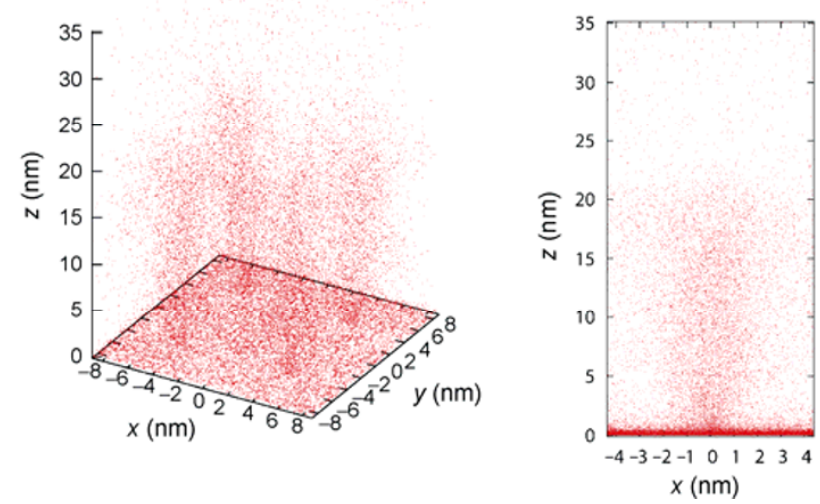

Fig. 5 Counterion distributions of salt-free solution with surface charge. $\xi=4.0$ and $D=2 l_{x y}$.

\subsection{Effect of added salt on the structure of the brush}

In this section the effect of added salt is discussed. The average heights $h$ of polyions in solutions with and without added salt are plotted in Fig. 6. Two different added salt concentrations were simulated at different grafting densities due to the limits of calculation time. The figure clearly shows the decrease in the heights of the polyelectrolyte brushes on the addition of salts, a finding that is in qualitative agreement with the results of experiments on the conformational change in poly[(2-dimethylamino)ethyl methacrylate] chains grafted on a surface by quartz crystal microbalance studied by dissipation and surface plasmon resonance [26]. This phenomenon is due to the screening of local charges by the added salts.

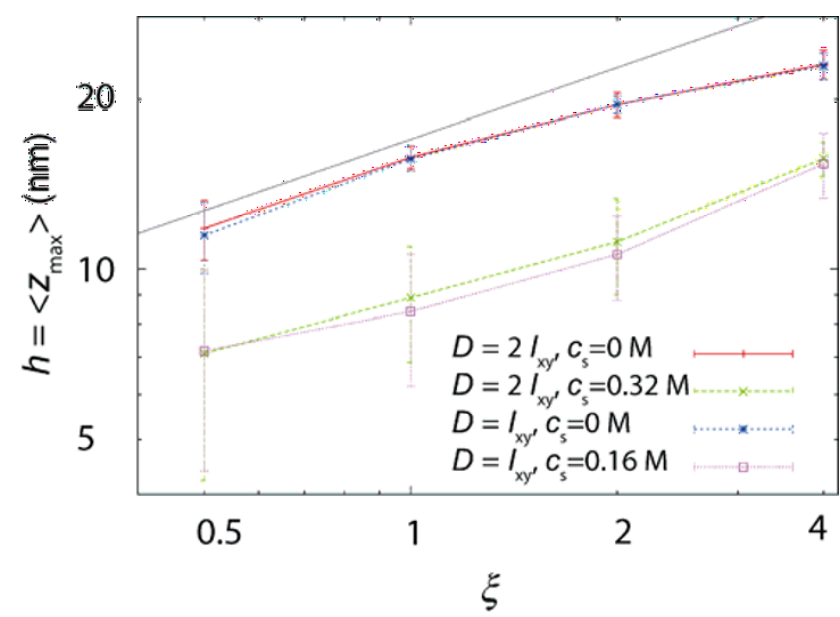

Fig. 6 Dependence of equilibrium average heights $h$ of polyions on linear charge densities $\xi$ with various salt concentrations. 
The straight line plotted in Fig. 6 also shows a power low fit in $h=\left\langle z_{\max }\right\rangle$ and the surface polymer spacing $D=l_{x y}$. According to the scaling theory, the addition of salt leads to the screening of the electrostatic interactions. In this strong screening regime, Debye screening is expected to reduce the counterion osmotic pressure responsible for the chain stretching. The height becomes $h \approx N a(a / D)^{2 / 3} \xi^{\alpha} \phi_{\mathrm{s}}^{-1 / 3}, \alpha=2 / 3$ [19], where $\phi_{\mathrm{s}}$ is the volume fraction of added salt. Our results do not show a dependence on the graft density and the added salt concentration. The fitting parameter of the power law is apparently less than $\alpha=2 / 3$ and this may be due to the length of the polyion under investigation. An oligomer of $N=128$ was used and this is too short for the polyion to entangle together to behave as an Alexander-de Gennes's neutral polymer. An interesting trend is found in the $\xi \geqslant 1$ region. In this region, $h$ shows a more marked increase than in the $\xi \leqslant 1$ region. This finding may also be related to counterion condensation. Snapshots of the equilibrium distribution of polyelectrolyte brush solutions are shown in Fig. 7. In comparison to Fig. 2, in both salt-free and added salt solutions, the counterions are condensed in the vicinity of the polyions when $\xi=4.0$. In the added
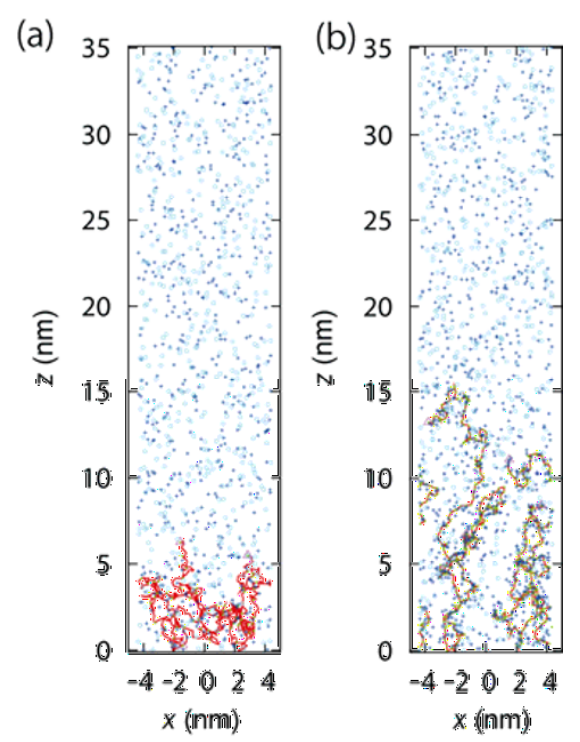

Fig. 7 Snapshots of polyelectrolyte brush solutions. (a) Added salt solution with $\xi=0.5$. (b) Added salt solution with $\xi=4.0$. $D=l_{x y}$. Red line, polyion; yellow/green circles, charge on polyion; blue cross, counterions; pink triangle, open end monomers, sky blue circles, anions. salt system with $\xi=0.5$ (Fig. 7(a)), where the average concentration of counterions seems higher in the polyion brush region than in the upper region, the counterions are not tied to the polyion. In the system with added salt, when $\xi=4.0$ (Fig. 7(b)), counterions that are apparently tied to the polyion are found. The additional stretching effect beyond the power law found in the $\xi \geqslant 1.0$ system cannot be explained by scaling or mean field theory and it may therefore be due to a dynamic effect called counterion polarization $[11,27]$, which has already been highlighted by Oosawa [24]. The existence of a condensed counterion layer causes a large dipole fluctuation in the longitudinal direction of the polyions and this causes the attractive force between two polyions. A more detailed analysis is required for further discussion of this phenomenon.

\subsection{Relation with adhesion and friction}

Before considering the friction, our simulation should be extended to treat the adhesion of the polyelectrolyte brush. According to the theoretical model for neutral brushes [28], the effect of chain length is critical for distances of less than $5 \mathrm{~nm}$. The height of the brush in this study is in the range above $8 \mathrm{~nm}$ and the chain length effect is therefore less critical for the adhesion force. When the Coulomb effect is considered, however, the effect of chain length for the adhesion is less clear.

Furthermore, improvements in the simulation are required to discuss the friction behavior in these systems, especially if the effect of solvent is included. Hydration lubrication [29] is important for understanding the friction behavior of polyelectrolyte brushes. Firstly, the solvent model was developed to include the solvation effect of polymers and counterand co-ions. In the framework of dissipative particle dynamics, the polarizability of a set of solvent molecules is described in terms of oscillators [30]. The solvent flow was included by calculating the Brownian particles by Langevin dynamics and the solvent flows by the Lattice Boltzmann method. In this method, the dynamics of a huge amount of small ions can be considered by treating each Brownian particle as a point described by a Stokes-source. In these methods ions are essentially treated as particles. To treat macroscopic phenomena, a multiphysical simulation based on continuum equations for ion solvent flow 
and electric fields was created. Distributions of small ions were treated by Nernst-Planck equations to consider transitional and nonbulk ion distributions [31]. Although these methods are under investigation, the friction of a more complicated tribo-system, which is governed by long-range Coulomb interactions, should be considered in the future.

\section{Conclusions}

Equilibrium structures of polyelectrolyte brushes grafted onto planes have been calculated by Monte Carlo Brownian dynamics simulations. In the case of a salt-free solution, scaling theories provided good predictions of the structure in the low $\xi$ region. However, in the high $\xi$ region additional shrinkage was found. The effect of surface charge showed not only the repulsion of the polyion from the surface in the low $\xi$ region but also the shrinkage in the high $\xi$ region due to the additional counterions added to achieve electrical neutrality. The addition of salts caused shrinkage of the brush heights and in the high $\xi$ region, additional extension was found. The relation between these findings and the adhesion and friction is discussed.

\section{Acknowledgment}

The present work was partially supported by the MEXT program "Elements Strategy Initiative to Form Core Research Center" (since 2012), MEXT; Ministry of Education Culture, Sports, Science and Technology, Japan.

Open Access: This article is distributed under the terms of the Creative Commons Attribution License which permits any use, distribution, and reproduction in any medium, provided the original author(s) and source are credited.

\section{References}

[1] Washizu H, Ohmori T. Molecular dynamics simulations of elastohydrodynamic lubrication oil film. Lubr Sci 22(8): 323-340 (2010)

[2] Washizu H, Sanda S, Hyodo S, Ohmori T, Nishino N, Suzuki
A. Molecular dynamics simulations of elasto-hydrodynamic lubrication and boundary lubrication for automotive tribology. J Phys Conf Ser 89: 12009 (2007)

[3] Washizu H, Kajita S, Tohyama M, Ohmori T, Nishino N, Teranishi H, Suzuki A. Mechanism of ultra low friction of multilayer graphene studied by coarse-grained molecular simulation. Faraday Discuss 156: 279-291 (2012)

[4] Kajita S, Washizu H, Ohmori T. Deep bulk atoms in a solid cause friction. Europhys Lett 87(6): 66002 (2009)

[5] Kajita S, Washizu H, Ohmori T. Approach of semi-infinite dynamic lattice Green's function and energy dissipation due to phonons in solid friction between commensurate surfaces. Phys Rev B 82(11): 115424 (2010)

[6] Kajita S, Washizu H, Ohmori T. Simulation of solid-friction dependence on number of surface atoms and theoretical approach for infinite number of atoms. Phys Rev B 86(7): 075453 (2012)

[7] Israelachvili J. Intermolecular and Surface Forces, 3d Ed. London: Academic Press, 2011.

[8] Raviv U, Giasson S, Kampf N, Gohy J F, Jerome R, Klein J. Lubrication by charged polymers. Nature 425(6954): 163-165 (2003)

[9] Gaisinskaya A, Ma L, Silbert G, Sorkin R, Tairy O, Goldberg R, Kampf N, Klein J. Hydration lubrication: Exploring a new paradigm. Faraday Discuss 156: 217-233 (2012)

[10] Kobayashi M, Terada M, Takahara A. Polyelectrolyte brushes: A novel stable lubrication system in aqueous conditions. Faraday Discuss 156: 403-412 (2012)

[11] Washizu H, Kikuchi K. Electric polarizability of DNA in aqueous salt solution. $J$ Phys Chem B 110(6): 2855-2861 (2006)

[12] Carrillo J-M Y, Brown W M, Dobrynin A V. Explicit solvent simulations of friction between brush layers of charged and neutral bottle-brush macromolecules. Macromolecules 45(21): 8880-8891 (2012)

[13] Goujon F, Ghoufi A, Malfreyt P, Tildesley D J. Frictional forces in polyelectrolyte brushes: Effects of sliding velocity, solvent quality and salt. Soft Matter 8(17): 4635-4644 (2012)

[14] Carrillo J-M Y, Russano D, Dobrynin A V. Friction between brush layers of charged and neutral bottle-brush macromolecules. Molecular dynamics simulations. Langmuir 27(23): 14599-14608 (2011)

[15] Kikuchi K, Yoshida M, Maekawa T, Watanabe H. Metropolis monte carlo method as a numerical technique to solve the fokker-planck equation. Chem Phys Lett 185: 335-338 (1991)

[16] Seror J, Merkher Y, Kampf N, Collinson L, Day A J, Maroudas A, Klein J. Normal and shear interactions between hyaluronan aggrecan complexes mimicking possible 
boundary lubricants in articular cartilage in synovial joints. Biomacromolecules 13(11): 3823-3832 (2012)

[17] Manning G S. Limiting laws and counterion condensation in polyelectrolyte solutions I. colligative properties. $J$ Chem Phys 51: 924-933 (1969)

[18] Saito M. Molecular dynamics simulations of proteins in water without the truncation of long-range coulomb interactions. Mol Simulation 8: 321-333 (1992)

[19] Pincus P. Colloid stabilization with grafted polyelectrolytes. Macromolecules 24: 2912-2919 (1991)

[20] Tran Y, Auroy P, Lee L-T. Determination of the structure of polyelectrolyte brushes. Macromolecules 32: 8952-8964 (1999)

[21] Hidetsugu Seki , Suzuki Y Y, Orland H. Self-consistent field study of polyelectrolyte brushes. J Phys Soc Jpn 76: 10461 (2007)

[22] Zhulina E B, Borisov O V, Birshtein T M. Structure of grafted polyelectrolyte layer. J Phys II France 2: 63-74 (1992)

[23] Ho Y-F, Shendruk T N, Slater G W, Hsiao P-Yi. Structure of polyelectrolyte brushes subject to normal electric fields. Langmuir 29: 2359-2370 (2013)

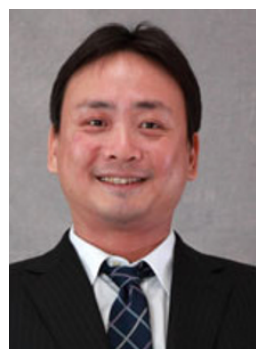

Hitoshi WASHIZU. He received his M.A. and Ph. D degrees in Physical Chemistry from the University of Tokyo, Japan, in 1998 and 2001 respectively. He joined Toyota Central R\&D Labs., Inc., from 2001. His current position is a leader of
[24] Oosawa F. Polyelectrolytes, Chapter 5. New York: CPC Press, 1971.

[25] Güven N. The crystal structures of 2M1 phengite and 2M1 muscovite. Z Kristallogr 134: 196-212 (1971)

[26] Wang X, Liu G, Zhang G. Conformational behavior of grafted weak polyelectrolyte chains: Effects of counterion condensation and nonelectrostatic anion adsorption. Langmuir 27(16): 9895-9901 (2011)

[27] Washizu H, Kikuchi K. Electrical polarizability of polyelectrolytes in salt-free aqueous solution. J Phys Chem B 106(43): 11329-11342 (2002)

[28] Guo L-Y, Zhao Y-P. Effect of chain length of self-assembled monolayers on adhesion force measurement by AFM. $J$ Adhes Sci Technol 20: 1281-1293 (2006)

[29] Klein J. Hydration lubrication. Friction 1(1): 1-23 (2013)

[30] Kinjo T, Yoshida H, Washizu H. Coarse-grained particle model for polar solvent. J Phys Soc Jpn Suppl, in press.

[31] Yoshida H, Kinjo T, Washizu H. Coupled lattice Boltzmann method for simulating electrokinetic flows in microchannels. In Proceedings of the 3rd European Conference on Microfluidics, 2012.

Washizu Research Group, Forintier Research Center of the TCRD. He is also a delegate of his research group in the Elements Strategy Initiative for Catalysts and Batteries, Kyoto University. His research areas cover computational chemistry of surfaces in tribology, polymer science, and electrochemistry. 